\title{
SENSIBILIDAD DEL CÁLCULO DE LOS NIVELES MEDIOS DEL MAR AL MÉTODO Y PERIODO DE LAS SERIES TEMPORALES DE LOS MAREÓGRAFOS EN LOS PROCESOS DE INUNDACIÓN: VALDELAGRANA (CÁDIZ)
}

\author{
Pablo Fraile Jurado ${ }^{1}$, Noela Sánchez-Carnero y José Ojeda Zújar ${ }^{1}$ \\ ${ }^{1}$ Departamento de Geografía Física y Análisis Geográfico Regional. Universidad de Sevilla \\ prraile@us.es, zujar@us.es \\ ${ }^{2}$ Kartenn Tecnologías para la Gestión Ambiental y Territorial \\ noela@kartenn.es
}

\section{RESUMEN}

En este trabajo se evalúa la importancia del método y periodo elegido en el cálculo del nivel medio del mar local y su necesaria corrección al cero topográfico para realizar análisis de vulnerabilidad de las áreas costeras ante potenciales inundaciones marinas. Para ello se evalúan dos métodos y diferentes periodos para el cálculo del nivel medio del mar a la serie temporal del mareógrafo de Cádiz. Cada uno de los valores obtenidos, una vez corregidos al cero topográfico, se proyectan sobre un MDE (obtenido a partir de datos adquiridos con un sensor LIDAR) sumándole una pleamar de coeficiente 1,17 para maximizar la expresión espacial de la inundación. Los resultados del análisis espacial de estas superficies de inundación muestran diferencias significativas y ponen de relieve la importancia de los parámetros del cálculo del nivel medio del mar en los trabajos de evaluación de inundaciones.

Palabras clave: series temporales, nivel medio del mar, MDE, inundación.

Fecha de recepción: mayo 2012.

Fecha de aceptación: marzo 2013. 


\section{ABSTRACT}

The aim of this work is to evaluate the significance of both the methodology and the period chosen for the calculation of the local mean sea level. Two methodologies and different periods of a time series of the tide gauge located in Cádiz, are evaluated. Each one of the resulting values are referred to the topographic datum, and projected over a DEM (obtained from Lidar data), adding a 1,17 coefficient high tide in order to maximize the spatial extension of the inundation. The obtained results show significant differences between the calculated mean sea levels. They remark the importance of considering appropriate parameters in the calculation of mean sea levels for estimating inundations.

Key words: time series, mean sea level, DEM, inundation.

\section{INTRODUCCIÓN}

Durante la última década, el cambio climático y sus efectos han pasado de ser una cuestión de importancia menor en la sociedad, de la que se ocupaba casi en exclusiva una pequeña parte de la comunidad científica, a ser entendido como el principal problema ambiental del planeta. La subida del nivel medio del mar ha sido considerada como una de las consecuencias más directas y evidentes del calentamiento global (IPCC, 2001, 2007a, 2007b).

En este contexto, numerosas instituciones nacionales y supranacionales (Naciones Unidas, Unión Europea, Environmental Protection Agency, Ministerio de Medio Ambiente) han elaborado diferentes estrategias y planes con el objetivo de identificar, valorar y prevenir los efectos potenciales derivados de la subida del nivel medio del mar. Algunos de estos trabajos han señalado la importancia de ir más allá de los cálculos globales y elaborar estudios regionales y locales que evalúen las consecuencias de una subida de nivel medio del mar a gran escala.

Dentro del contexto de los estudios de impactos asociados a la subida del nivel medio del mar se han publicado numerosos trabajos para la evaluación de zonas potencialmente vulnerables a inundaciones futuras (Titus y Richmann, 2000, Marfai y King, 2005, Zhang, 2011, Fraile et al. 2012a). Aunque se han aplicado diferentes procedimientos, la mayoría de estos trabajos coinciden en identificar como áreas inundables aquellas cuya altitud, definida por un modelo digital de elevaciones (MDE) quede por debajo de una cota de inundación futura determinada. Esta cota se calcula habitualmente por la adición de la subida del nivel medio del mar esperada para el futuro (calculada a partir de modelos numéricos de cambio climático) a la cota del cero topográfico de referencia. En síntesis, todos coinciden metodológicamente en la necesidad de modelar dos superficies:

- La superficie topográfica (generalmente a través de MDE) referida al datum altimétrico oficial, que en el caso de España es el nivel medio del mar en Alicante (NMMA).

- La superficie del mar correspondiente al nivel medio del mar local (NMML).

El NMMA constituye la referencia altimétrica (cota 0) de los mapas topográficos y de los MDEs de la España peninsular. Sin embargo este valor no coincide necesariamente 
con el nivel medio del mar en cualquier otro punto de la costa (NMML). Este desfase provoca diferencias en los cálculos de cotas de inundación futuras (en su valor vertical) que conllevan diferencias muy significativas en el cálculo de las áreas inundadas (una pequeña diferencia de altitud puede representar grandes diferencias en la horizontal en zonas llanas).

Por esta razón, tanto para estos trabajos, centrados en la evaluación de las consecuencias de una subida del nivel medio del mar, como para aquellos centrados en los efectos de los eventos extremos marinos (temporales, tsunamis), la altura del NMML es un concepto fundamental al que se debe prestar atención. Se define como la diferencia vertical entre un valor local (generalmente un promedio) obtenido de los registros del mareógrafo y el NMMA. Es decir, mide la altitud que debe añadirse o sustraerse al cero de los mapas topográficos para obtener las zonas inundables. La principal diferencia de este último es su carácter local y, por lo tanto, variable espacialmente.

Para el cálculo del NMML se utilizan las series temporales de los mareógrafos. Desde el punto de vista climático se considera que el cálculo de un valor medio a partir de una serie temporal requiere de un mínimo de 30 años de registros (Cuadrat y Pita 1997; OMM, 2010). Sin embargo, esto no es siempre posible en los mareógrafos españoles, donde por ejemplo la Red de Mareógrafos de Puertos del Estado (REDMAR) se estableció en 1992, por lo que los autores se ven obligados a utilizar periodos menores. La red del IEO posee mareógrafos con series más largas.

En los trabajos publicados sobre evaluación de inundaciones asociadas a la subida del nivel medio del mar el periodo y método utilizados para el cálculo del NMML han sido variables (Wyrtki y Mitchum, 1990; Woodworth et. al, 1999; Woodworth et. al, 2009). Sin embargo, en estos trabajos estos parámetros se han escogido sin someterlos a discusión, y no es frecuente la publicación de estudios que aborden la relevancia de la elección del método y periodo en el cálculo de esta variable.

Durante las próximas décadas es esperable que los trabajos de análisis de áreas inundables por la subida del nivel medio del mar incrementen su importancia en la planificación urbanística y ordenación del territorio, ya que permiten delimitar áreas especialmente sensibles (playas, puertos, espacios naturales protegidos costeros...) ante una previsible situación de cambio climático y subida del nivel medio del mar. Por este motivo es fundamental mejorar la precisión de los cálculos realizados en estos trabajos y disminuir la variabilidad/ aleatoriedad asociada a los mismos. Este trabajo pretende señalar la relevancia de la elección de los parámetros implicados en el cálculo del NMML.

\section{OBJETIVO Y ÁREA DE ESTUDIO}

El objetivo de este trabajo es evaluar la sensibilidad del cálculo de las aéreas inundadas al uso de diferentes métodos y periodos en el cálculo del nivel medio del mar local (NMML).

El área de estudio seleccionada para este trabajo comprende el espacio de marismas situado entre la playa de Valdelagrana y la orilla derecha del río San Pedro, el municipio de El Puerto de Santa María (Cádiz) (Figura 1). La elección de esta zona responde a su representatividad en relación a otras muchas áreas costeras asociadas a marismas mareales y a la disponibilidad de datos adecuados para la metodología del trabajo. 


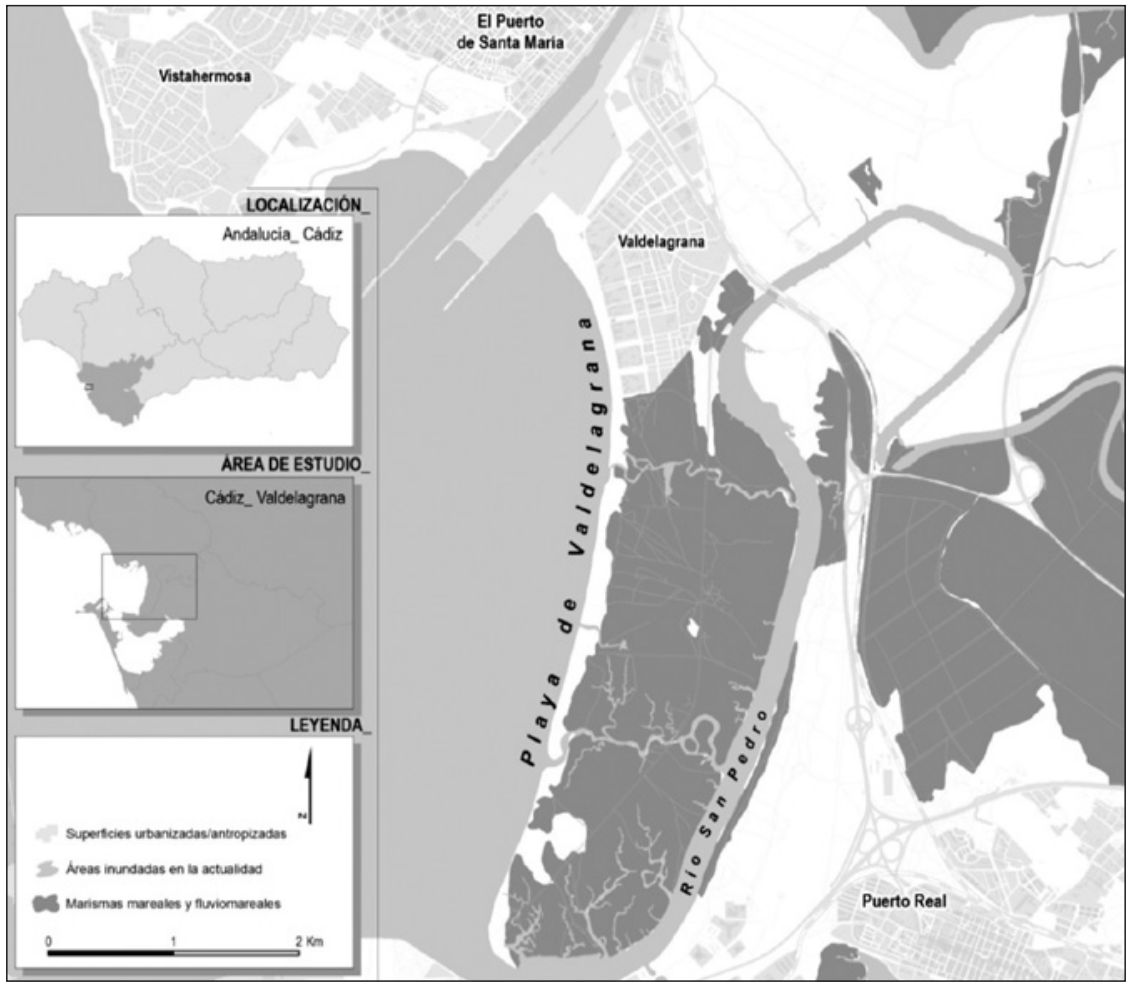

Por un lado, el área de estudio cuenta con un mareógrafo (mareógrafo de Cádiz) situado a una distancia menor a $5 \mathrm{~km}$, lo que permite asumir los valores de tendencia del dispositivo sin necesidad de aplicar procesos de interpolación espacial (Fraile, 2011).

Por otro lado, este mareógrafo presenta una elevada tasa de cambio del nivel medio del mar (4,1 mm/año, Fraile (2011)). En un mareógrafo con una tasa de cambio próxima a cero, la variación del valor del nivel medio del mar sería mínima, por lo que la comparación de métodos no tendría sentido. Por otro lado, la elevada tasa de cambio de este mareógrafo nos permite maximizar las diferencias encontradas en el valor del nivel medio del mar al considerar distintos periodos para su cálculo (objetivo de estudio de este trabajo). Esta tasa podría explicarse, en parte, por la coincidencia de una subida absoluta del nivel medio del mar (3,1 mm / año durante el periodo 1992-2011 de acuerdo con los datos aportados por satélites altimétricos para la superficie marina del Golfo de Cádiz) y por los efectos de la subsidencia del área de estudio, evaluada en -2 mm / año por Ruttigliano et al. (2005) en estudios de periodo corto (Fraile et al., 2012b).

Por último, en esta zona se cuenta con un MDE de elevada precisión horizontal y vertical (derivado de datos LIDAR), lo que es imprescindible para la correcta observación de las inundaciones derivadas de los distintos niveles medios del mar calculados. 


\section{FUENTES Y METODOLOGÍA}

\subsection{Fuentes}

Para la elaboración de este trabajo se han empleado dos tipos de datos:

- Datos altimétricos sobre la superficie marina: valores medios mensuales del nivel del mar local en el mareógrafo de Cádiz (fig. 2), procedentes del Permanent Service for Mean Sea Level (PSMSL). Este servicio recoge los registros proporcionados por el instituto Español de Oceanografía (IEO) para el mareógrafo de Cádiz, encargado de su gestión y mantenimiento. Estos valores son publicados en formato METRIC, es decir, medidos a partir del cero del mareógrafo. Existen datos sobre esta serie desde enero de 1961 hasta diciembre de 2009. Adicionalmente, y para mejorar la representación espacial, se utilizaron los valores locales de alturas de la pleamar obtenidos en la web de Puertos del Estado (www.puertos.es), procedentes del modelo NivMar para el periodo 2007-2017, y contrastadas con otras publicaciones (Bruno y Tejedor, 1996).

- Datos altimétricos sobre la superficie emergida: MDE derivado de datos LIDAR obtenidos en una campaña realizada en 2009, con una resolución espacial de 1 metro y una precisión vertical estimada en $15 \mathrm{~cm}$ (Thieler 2009). Los valores altimétricos están referenciados al Nivel del Mar en Alicante (NMMA).

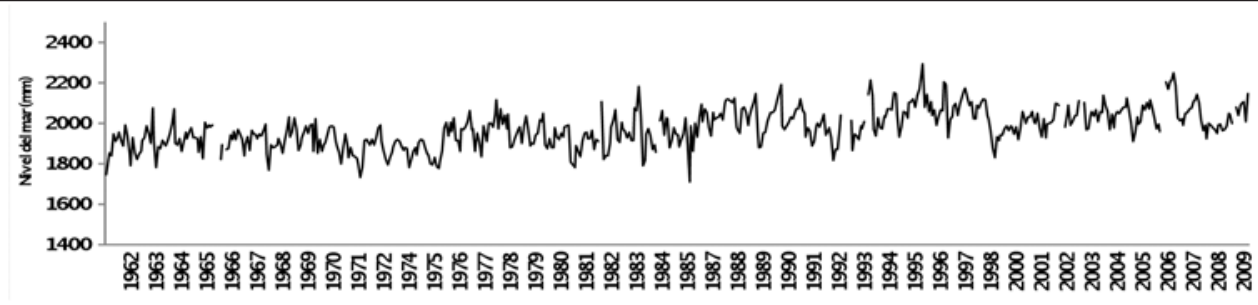

\subsection{Métodos}

Para el cálculo de los NMML se han empleado dos tipos de métodos: i) métodos basados en el cálculo del promedio de la serie temporal durante diferentes periodos, y ii) métodos basados en el cálculo de la posición para el momento «presente» en la recta de regresión lineal de la serie temporal del mareógrafo. Por último, en este apartado se sintetizan los métodos de evaluación de las áreas inundadas empleados.

\subsubsection{La influencia de la duración de la serie temporal}

Con la intención de evaluar la importancia de la duración de la serie temporal en el cálculo del NMML, se calcularon los valores medios para tres periodos (M1, M2 y M3) 
utilizando el promedio de la serie temporal en cada uno de ellos. De este modo puede comprobarse si la tasa de cambio del nivel del mar de ese mareógrafo muestra resultados significativamente distintos en función del periodo elegido.

Los periodos utilizados correspondieron: i) a toda la serie temporal disponible, desde el establecimiento del mareógrafo en 1961 hasta el final de la serie en 2009 (M1), que permite disponer de un periodo de más de 30 años en el que se ha detectado una tendencia estadística (Fraile, 2011); ii) al periodo de referencia utilizado por el IPCC (2007) para proyectar los niveles del mar en el futuro (M2); y iii) al periodo definido a partir del inicio de las mediciones de los satélites altimétricos, que comenzaron en julio de 1992 (M3), coincidente además con la mayor parte de los mareógrafos de la red de Puertos del Estado, lo que permite contrastar cualquier resultados con los publicados acerca de las variaciones locales del nivel medio del mar tanto a escala global (IPCC 2007) como nacional (Tabla 1).

Tabla 1

MÉTODOS Y PERIODOS OBTENIDOS DEL ANÁLISIS DE LAS DIFERENTES SERIES

TEMPORALES DEL MAREÓGRAFO DE CÁDIZ

\begin{tabular}{|c|c|c|}
\hline NIVEL & MÉTODO & PERIODO \\
\hline M1 & Media aritmética & $1961-2009$ \\
\hline M2 & Media aritmética & $1990-1999$ \\
\hline M3 & Media aritmética & $1992-2009$ \\
\hline R1 & Regresión lineal & $1961-2009$ \\
\hline R3 & Regresión lineal & $1992-2009$ \\
\hline
\end{tabular}

\subsubsection{La influencia del método utilizado}

Para identificar la sensibilidad del cálculo del NMML al método empleado, se calcularon dos NMML mediante un análisis de regresión lineal, en el que el valor del NMML es el correspondiente al de la posición de la recta de regresión lineal obtenida para el último año del periodo. Con la intención de compararlos con los resultados obtenidos en el análisis del apartado anterior, se calcularon los valores R1 y R3, correspondientes a los mismos periodos que M1 y M3 respectivamente (Tabla 1). El valor correspondiente al periodo 1990-1999 (el mismo que M2) no se incluye en el análisis por tratarse de una serie temporal demasiado corta (9 años) como para permitir un análisis de regresión fiable para la obtención de su tendencia.

\subsubsection{Método de análisis espacial del área inundable}

Para evaluar la sensibilidad de la simulación de inundaciones, sobre un MDE derivado de datos LIDAR, al uso de diferentes métodos y periodos en el cálculo del NMML, cada uno de los valores de altura calculados (NMML) fueron proyectados sobre el MDE, de manera que se observase cómo las diferencias verticales se comportan en la proyección horizontal de las áreas inundables. 
Para realizar esta proyección de forma correcta es necesario llevar a cabo previamente la corrección del datum altimétrico de los NMML calculados para los diferentes periodos y métodos, puesto que el MDE empleado (y en general, cualquier MDE o mapa topográfico) utiliza como valor de referencia el NMMA. En nuestro caso, los NMML están medidos desde el denominado «cero del mareógrafo», que se corresponde con el valor teórico más bajo que puede alcanzar el nivel del mar por causas astronómicas, y que en el mareógrafo de estudio se encuentra 1,885 m por debajo del NMMA. Este valor se sustrajo a los cuatro NMML obtenidos, resultando así los niveles medios del mar referenciados al NMMA (NMMLR) y, por lo tanto, comparables con el MDE Lidar (Tabla 2).

A la hora de proyectar los NMMLR sobre el MDE mediante el método descrito, es preciso resolver dos problemas:

i) con la proyección de los valores de NMMLR se obtendrían las áreas de inundación en una posición del nivel medio del mar en la actualidad, difícilmente observables en un MDE de la zona emergida, al estar situados prácticamente en la zona media de la franja intermareal.

ii) Por limitaciones asociadas al método de adquisición de los datos, la altura mínima del MDE empleado es de 0,47 metros, por lo que se imposibilitaría la representación de los datos de los NMMLR de la tabla 1, al quedar todos por debajo de esta altitud.

Por estos dos motivos, se añadió a todas las alturas calculadas la mitad del rango mareal (3,4 metros) correspondiente al día 19 de marzo de 2011, en el que hubo una marea de coeficiente 1,17 , muy cercano al máximo teórico posible (o marea astronómica).

Aunque la simulación del alcance de las áreas inundadas por una pleamar requiere de la consideración de elementos como el tipo de la superficie inundada (vegetada, suelo desnudo, etc.), la percolación o la propagación de la propia onda de marea por los caños mareales, en este trabajo se ha simplificado el proceso obviando los puntos anteriores y considerando la inundación como la consecuencia del avance de una masa de agua plana de altura igual al NMMLR (modelo bathtub).

Con la intención de evaluar la importancia de la corrección altimétrica local, se representó también un NMML, denominado L0, correspondiente a la altura de la misma pleamar sin haber realizado ninguna corrección vertical.

\section{RESULTADOS}

La importancia del método de cálculo utilizado para obtener el valor del NMML es evidente al comparar los resultados obtenidos para toda la serie temporal (M1 y R1) (figura 3). Considerando el mismo periodo se obtienen alturas con más de $9 \mathrm{~cm}$ de diferencia vertical (tabla 2), siendo la regresión lineal el método que obtiene valores más altos $(0.187 \mathrm{~m})$. Este comportamiento responde, evidentemente, a la existencia de una tendencia positiva, que es compensada cuando se utilizan métodos como el cálculo de la media.

La diferencia observada entre ambos métodos no es, sin embargo, constante. En los valores de M3 y R3 (correspondientes ambos al mismo periodo, 1992-2009), aunque R3 sigue siendo más alto que M3 $(17 \mathrm{~cm}$ frente a $15.5 \mathrm{~cm})$, las diferencias se acortan significativa- 
mente (de $9 \mathrm{~cm}$ a $1.5 \mathrm{~cm}$ ). Este es el resultado esperado por el método utilizado en el cálculo del valor de la regresión (valor del punto más actual). En este sentido, cuanto más cerca al punto final de la serie y más corto sea el periodo seleccionado, menor será la diferencia entre ambos métodos.

Con respecto a los periodos estudiados, cuando utilizamos métodos de tendencia central, como la media, los valores de NMML obtenidos aumentan cuanto más se acerca el periodo al final de la serie, es decir, a la zona de registros con valores más altos (serie con tendencia positiva). Este efecto se observa incluso cuando el periodo es mayor en amplitud (M3 frente a M2).

Los métodos de regresión, por su parte, son sensibles a la localización del periodo observado dentro de la serie completa (valor medio del periodo) y a las características de la serie en ese periodo (tendencia). En nuestro caso de estudio el periodo R3, pertenece a una zona de la serie donde el crecimiento de la misma es menor que el de la serie general (2,1 mm/año frente a 4,1 mm/año) y, por lo tanto, el valor de NMML será menor que R1.

Tabla 2

RESULTADOS OBTENIDOS EN EL CÁLCULO DE LOS NIVELES MEDIO DEL MAR MEDIANTE EL PROMEDIO DE LA SERIE TEMPORAL Y EL ANÁLISIS DE REGRESIÓN. NMML: NIVEL MEDIO DEL MAR LOCAL REFERIDO AL CERO DEL MAREÓGRAFO; NMMLR: NIVEL MEDIO DEL MAR LOCAL REFERIDO AL NMMA

\begin{tabular}{|c|c|c|c|}
\hline NIVEL & PERIODO & NMML (m) & NMMLR (m) \\
\hline M1 & $1961-2009$ & 1,977 & 0,092 \\
\hline M2 & $1990-1999$ & 2,031 & 0,146 \\
\hline M3 & $1992-2009$ & 2,04 & 0,155 \\
\hline R1 & $1961-2009$ & 2,072 & 0,187 \\
\hline R3 & $1992-2009$ & 2,055 & 0,17 \\
\hline H0 & - & - & 0 \\
\hline
\end{tabular}

Tabla 3

RESULTADOS OBTENIDOS EN LA SIMULACIÓN DE INUNDACIONES EN CADA NIVEL CALCULADO, EN TOTAL, EN INCREMENTOS ABSOLUTOS CON RESPECTO AL NIVEL HO (INC. AB.) Y EN INCREMENTO RELATIVO CON RESPECTO A HO (INC. REL.)

\begin{tabular}{|c|c|c|c|c|}
\cline { 2 - 5 } \multicolumn{1}{c|}{} & PERIODO & TOTAL (ha.) & INC. AB. (ha.) & INC. REL. (\%) \\
\hline H0 & - & 490,87 & - & \multicolumn{1}{|c|}{} \\
\hline M1 & $1961-2009$ & 662,68 & 171,81 & 35,00 \\
\hline M2 & $1990-1999$ & 699,7 & 208,83 & 42,54 \\
\hline M3 & $1992-2009$ & 705,78 & 214,91 & 43,78 \\
\hline R1 & $1961-2009$ & 780,11 & 289,24 & 58,92 \\
\hline R3 & $1992-2009$ & 727,44 & 236,57 & 48,19 \\
\hline
\end{tabular}


Figura 3

DIAGRAMA DE LAS ALTURAS DE LOS NMMLR CALCULADOS EN FUNCIÓN DE SU DURACIÓN Y MÉTODO

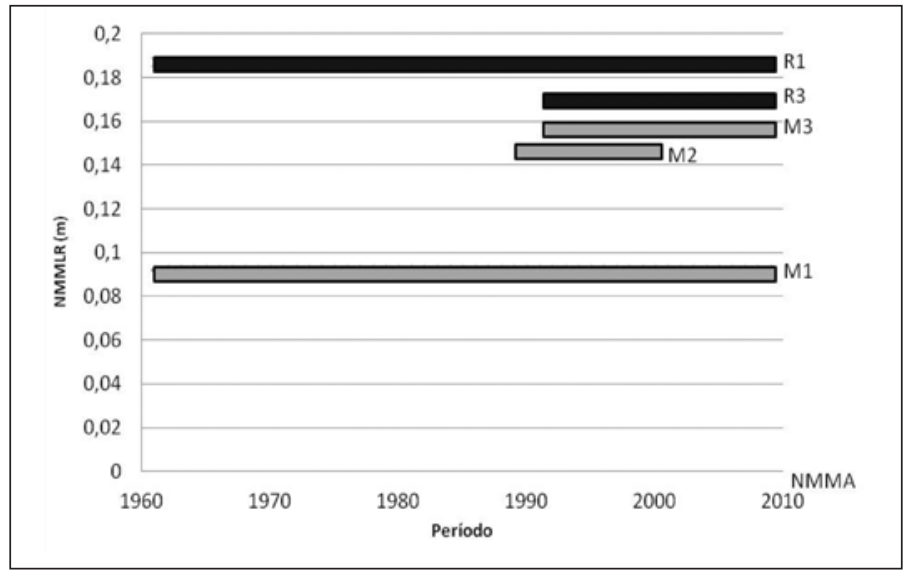

Figura 4

INUNDACIONES BAJO CONDICIONES DE COEFICIENTE DE MAREA 1,17 CORRESPONDIENTES A LOS PERIODOS M1 YR4 EN EL SECTOR CENTRAL DEL ÁREA DE ESTUDIO

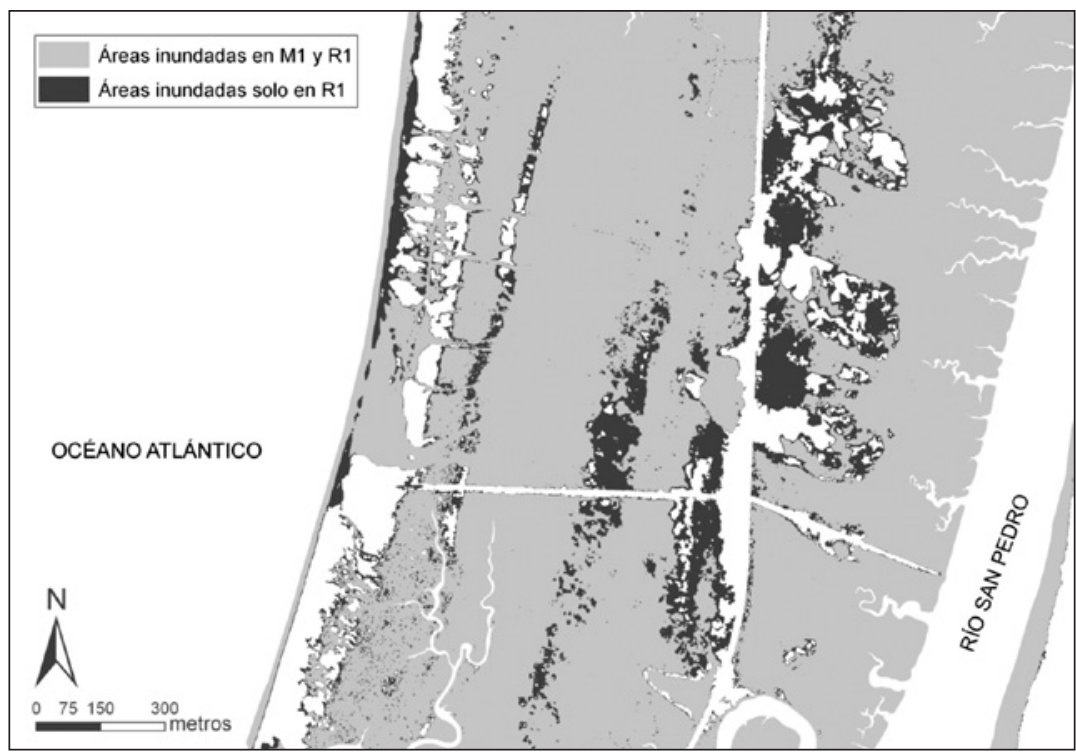

Todas las áreas de inundación generadas mostraron diferencias relevantes con respecto a las calculadas con el nivel de referencia H0 (Tabla 3). Aunque las diferencias observadas para los distintos NMMLR calculados tienen un rango inferior a $10 \mathrm{~cm}$, la simulación de las inundaciones producidas por cada uno de ellos en un área topográficamente baja y morfológicamente llana, como el área de estudio, muestra resultados notablemente diferentes (Tabla 3). 
Las figuras 4 y 5 muestran diferentes escenarios de inundación, de acuerdo a los NMML calculados anteriormente. Las espacialización de las diferencias verticales obtenidas entre distintos métodos (figura 4) y para distintos periodos (figura 5), dejan ver importantes diferencias en cuanto a las superficies inundadas (tabla 3). Por ejemplo, la diferencia de $9 \mathrm{~cm}$ observada en los NMML calculados para R1 y M1, da lugar a una diferencia sobre el terreno de 66 ha más inundadas en R1 (un 7,8\% del total de la marisma, incluyendo caños mareales).

Figura 5

INUNDACIONES BAJO CONDICIONES DE COEFICIENTE DE MAREA 1,17 CORRESPONDIENTES A LOS NIVELES HO, $M 1, M 2 Y M 3$

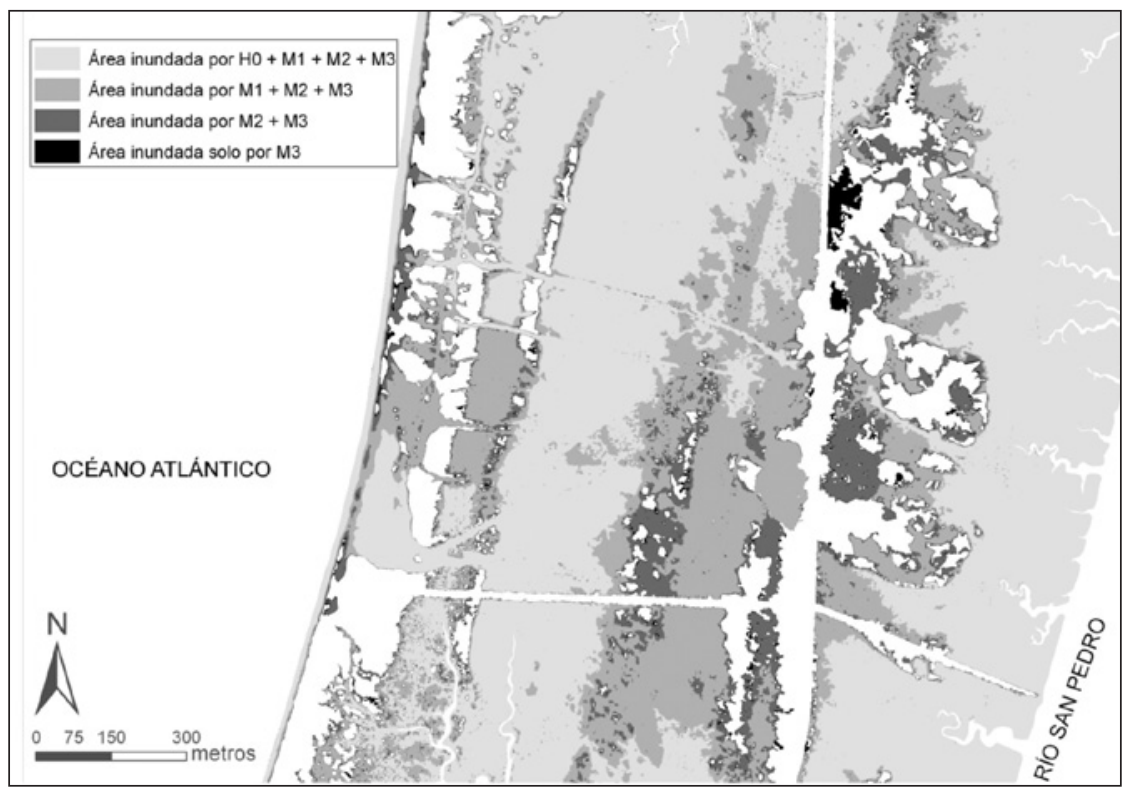

Resumiendo, cuando se trabaja con una serie con tendencia positiva (o negativa), los métodos de regresión siempre aportarán valores mayores (o menores) de NMML que aquellos obtenidos por métodos de suavizado, como la media. En estos casos, cuanto mayor sea el periodo utilizado, mayor será el rango de valores de altura del NMML y, por lo tanto, menores los valores de NMML calculados (y mayores las diferencias con respecto a los métodos de regresión).

\section{CONCLUSIONES Y DISCUSIÓN}

Los resultados obtenidos en este trabajo muestran la sensibilidad de las medidas de NMML tanto al periodo considerado como al método utilizado para su cálculo, cuando se trabaja con una serie temporal con tendencia. La magnitud de las diferencias observadas son del orden de los cálculos de subida para los próximos 50 años realizados por el IPCC (2007) lo que destaca la relevancia de la consideración de estos parámetros a la hora de abordar un estudio de inundación. 
Esto introduce una nueva problemática en el ya complejo cálculo de los escenarios de inundación futuros asociados a la subida del nivel medio del mar y en los estudios de riesgos asociados a los mismos. Estos parámetros, periodo y método, han sido hasta el momento definidas por organismos internacionales o en trabajos científicos concretos (Titus y Narayan, 1998; IPCC, 2001; IPCC, 2007), pero no han sido ampliamente discutidos por la comunidad científica, y es común que ni si quiera se mencionen sus valores explícitamente en muchos trabajos (Thieler, 2009, Hinkel, 2010, Zhang, 2010).

Este trabajo pone de manifiesto i) la necesidad de referenciar los futuros análisis de subida del nivel del mar al método y periodo utilizados para calcular el NMML, para que sus resultados puedan ser adecuadamente interpretados y, ii) la necesidad de abordar los estudios de inundaciones futuras (o estudios asociados a estos) a partir de un análisis previo de la variabilidad de los valores de NMML que se obtienen a partir de la serie temporal utilizada; lo que permite definir los casos más extremos que se extraen de los valores NMML y discutir los resultados.

\section{BIBLIOGRAFÍA}

CUADRAT, J.M. y PITA, M.F. (1997): Climatología, Cátedra.

INTERGOVERNMENTAL PANEL ON CLIMATE CHANGE (IPCC) (2001): The scientific basis. Cambridge University Press, Cambridge.

INTERGOVERNMENTAL PANEL ON CLIMATE CHANGE (IPCC) (2007): Cambio climático 2007 Impactos, adaptación y vulnerabilidad. Cambridge University Press.

INTERGOVERNMENTAL PANEL ON CLIMATE CHANGE (IPCC) (2007): Cambio climático 2007 Bases de Ciencia Física. Cambridge University Press.

HINKEL, J., y KLEIN, R. (2009): «The DINAS-COAST project: developing a tool for the dynamic and interactive assessment of coastal vulnerabilty», Global environmental change, 19.

FRAILE JURADO, P. (2011): Análisis de la problemáticas asociadas a la espacialización, evolución y representación de niveles del mar presentes y futuros en Andalucía, Tesis Doctoral, Universidad de Sevilla.

FRAILE JURADO, P. y OJEDA ZÚJAR, J. (2012a): «Evaluación de la peligrosidad asociada al aumento de la superficie inundable por la subida del nivel medio del mar en la costa entre Cádiz y Tarifa», Geofocus, vol. 12, 329-348.

FRAILE JURADO, P. y OJEDA ZÚJAR, J. (2012b): «The importance of the vertical accuracy of digital elevation models in gauging inundation by sea level rise along the Valdelagrana beach and marshes (Bay of Cádiz, SW Spain)», Geomarine Letters, DOI: 10.1007/ s00367-012-0317-8.

MARFAY, M. y KING, L. (2007): «Monitoring land subsidence in Semarang (Indonesia)», Environmental Geology, vol. 53, 3, pp. 651-659.

PERIÁÑEZ, R. (2010): Fundamentos de Oceanografía Dinámica, Secretariado de Publicaciones de la Universidad de Sevilla.

PUGH, D. (2004): Changing sea levels, Cambridge University Press.

POKHAREL, P., TAKEDA, M. y MATSUO N. (2007): «Study on Inundation in the Coastal City Due to Heavy Rainfall Considering the Effect of Global Warming», en World Environmental and Water Resources Congress. 
RUTIGLIANO, P., C. FERRARO, R. DEVOTTI, R. LANOTTE, V. LUCERI, A. NARDI, R. PACIONE, C. SCIARRETTA, C. DOGLIONI, E. GUEGUEN y F. VESPE (2000): «Vertical motions in the Western Mediterranean area from geodetic and geological data» en The tenth General Assembly of the WEGENER Project.

TEJEDOR MARTÍNEZ, L., y BRUNO MEJÍAS, M. (1996): «Niveles del mar en la Bahía de Cádiz», en Estudios para la ordenación, planificación y gestión integrada de las zonas húmedas de la Bahía de Cádiz, Oikos Tau.

TEL, E., y GARCÍA, M (2004): «Nivel del mar en las costas españolas y su relación con el clima», comunicación en el III Congreso de la Asociación Española de Climatología, Santander.

THIELER, R. (2009): Coastal sensitivity to sea level rise: a Focus on the Mid-Atlantic region, USGS.

TITUS, J. y NARAYAN, V. (1998): The probability of sea level rise, EPA, Washington.

TITUS, J. y RICHMANN, C. (2001): Maps of Lands Vulnerable to Sea Level Rise: Modeled Elevations along the U.S. Atlantic y Gulf Coasts, Environmental Protection Agency.

WADE, T., y SOMMER, S. (2006): A to Z GIS, ESRI.

WOODWORTH, P. L., TSIMPLIS, M. N., FLATHER, R. A. y SHENNAN, I. (1999), «A review of the trends observed in British Isles mean sea level data measured by tide gauges», Geophysical Journal International, 136: 651-670.

WOODWORTH, P., TEFERLE, F., BINGLEY, R., SHENNAN, I. y WILLIAMS, S. (2009): «Trends in UK mean sea level revisited», Geophysical Journal International, 176: 19-30.

WYRTKI, K., y G. MITCHUM (1990): «Interannual Differences of Geosat Altimeter Heights and Sea Level: The Importance of a Datum», Journal of Geophysical Research, 95(C3), 2969-2975.

ZHANG K. (2011): Analysis of Non-Linear Inundation from Sea Level rise Using Lidar Data: A Case Study for South Florida, Climatic Change, vol. 106, 4, pp. 535-545.

\section{AGRADECIMIENTOS}

Esta investigación forma parte del desarrollo del proyecto de excelencia de la Junta de Andalucía (RNM-6207) Espacialización y Difusion Web de Datos de Urbanización, y Fitodiversidad para el Análisis de Vulnerabilidad ante los Procesos de Inundación Asociados a la Subida del Nivel del Mar en la Costa Andaluza, así como del proyecto Espacialización y Difusión Web de Variables Demográficas, Turísticas y Ambientales para la Evaluación de la Vulnerabilidad Asociada a la Erosión de Playas en la Costa Andaluza (CSO2010-15807) financiado por el Ministerio de Ciencia e Innovación y por Fondos Feder. 